Revue internationale P.M.E.

Économie et gestion de la petite et moyenne entreprise

\title{
La structure d'endettement des PME françaises: une étude sur données de panel
}

\section{Ydriss Ziane}

Volume 17, numéro 1, 2004

URI : https://id.erudit.org/iderudit/1008454ar

DOI : https://doi.org/10.7202/1008454ar

Aller au sommaire du numéro

Éditeur(s)

Presses de l’Université du Québec

ISSN

0776-5436 (imprimé)

1918-9699 (numérique)

Découvrir la revue

Citer cette note

Ziane, Y. (2004). La structure d'endettement des PME françaises: une étude sur données de panel. Revue internationale P.M.E., 17(1), 123-138.

https://doi.org/10.7202/1008454ar
Résumé de l'article

Cette contribution présente les résultats de tests d'hypothèses relatifs aux théories financières de la structure d'endettement des entreprises mettant l'accent sur la fiscalité et le risque de défaut ainsi que les coûts d'agence et l'asymétrie d'information. Nous testons, par le biais d'un modèle économétrique dynamique, une grille d'hypothèses basée sur la prise en compte de ces imperfections du marché du financement. L'échantillon considéré se compose de $2880 \mathrm{PME}$ françaises observées durant la période 1993-2000. Nos résultats révèlent que les niveaux d'endettement, liés positivement aux variables de tangibilité des actifs et de croissance et négativement à la rentabilité, varient sensiblement en fonction de la taille des firmes. Ainsi, le seuil de 20 salariés apparaît comme stratégique dans l'étude des comportements financiers des petites et moyennes entreprises françaises.
Ce document est protégé par la loi sur le droit d'auteur. L’utilisation des services d’Érudit (y compris la reproduction) est assujettie à sa politique d'utilisation que vous pouvez consulter en ligne.

https://apropos.erudit.org/fr/usagers/politique-dutilisation/ 


\title{
La structure d'endettement des PME françaises: une étude sur données de panel
}

\author{
Ydriss ZIANE \\ Université de Paris $X$, Nanterre
}

\author{
MOTS CLÉS
}

\section{Structure financière - Endettement - Coûts d'agence \\ Asymétrie de l'information - Données de panel Modèle dynamique}

\begin{abstract}
RÉSUMÉ
Cette contribution présente les résultats de tests d'hypothèses relatifs aux théories financières de la structure d'endettement des entreprises mettant l'accent sur la fiscalité et le risque de défaut ainsi que les coûts d'agence et l'asymétrie d'information. Nous testons, par le biais d'un modèle économétrique dynamique, une grille d'hypothèses basée sur la prise en compte de ces imperfections du marché du financement. L'échantillon considéré se compose de 2880 PME françaises observées durant la période 1993-2000. Nos résultats révèlent que les niveaux d'endettement, liés positivement aux variables de tangibilité des actifs et de croissance et négativement à la rentabilité, varient sensiblement en fonction de la taille des firmes. Ainsi, le seuil de 20 salariés apparaît comme stratégique dans l'étude des comportements financiers des petites et moyennes entreprises françaises.
\end{abstract}

\section{L'AUTEUR}

YDRISS ZIANE est attaché temporaire d'enseignement et de recherche (ATER) à l'Université de Paris X-Nanterre où il enseigne l'économie monétaire et financière. Il est membre du MODEM (Modélisation de la dynamique économique et monétaire) où il mène une recherche doctorale sur les déterminants de la structure financière de PME. Adresse : Ydriss Ziane, MODEM, Bât. G, Université Paris X-Nanterre, 200, avenue de la République, 92001 Nanterre, Cédex, France. Courriel : <yziane@u-paris10.fr>. 


\begin{abstract}
In this paper, we present results from empirical tests about financial theories of corporate leverage relative to taxes and bankruptcy costs, agency problems and asymmetric information. We use a dynamic panel data model to test a set of hypotheses linked to the existing of these market imperfections. The sample is formed by 2880 small and medium-sized French companies observed during the period 1993-2000. Our results suggest that financial leverage, positively linked to asset tangibility and growth and negatively to profitability, is very dependant from firm's size measured in terms of manpower. Hence, the threshold of 20 employees appears as determinant in leverage financial strategies maintaining by French SME.
\end{abstract}

\title{
RESUMEN
}

Esta contribución presenta los resultados de pruebas empíricas de hipótesis relativos a las teorías financieras de la estructura de endeudamiento de las empresas en relación con la fiscalidad y el riesgo de defecto así como los costes de agencia y la asimetría de información. Probamos, por la inclinación de un modelo econométrico de datos de panel dinámico, una rejilla de hipótesis basada en la consideración de estas imperfecciones del mercado de la financiación. La muestra en cuestión consta de 2880 PyMEs francesas observadas durante el período 1993-2000. Nuestros resultados sugieren que los niveles de endeudamiento, vinculados positivamente con las variables de tangibilidad de los activos y de crecimiento y negativamente con la rentabilidad, varían sensiblemente en función del tamaño de las empresas. Así, el umbral de 20 empleados aparece como estratégica en el estudio de los comportamientos financieros de las pequeñas y medianas empresas francesas.

\section{ZUSAMMENFASSUNG}

Dieser Beitrag präsentiert die Testergebnisse von Hypothesen betreffend den finanziellen Theorien der Verschuldungsstruktur mit dem Schwerpunkt der Besteuerung, dem Risikoverhalten sowie der Informationsasymetrie. Mit einem dynamischen, ökonomischen Modell, testeten wir ein Hypothesengitter, das auf der Berücksichtigung der Mängel des Finanzierungsmarktes aufbaut. Die Stichprobe setzt sich aus 2.880 französischen KMU zusammen, die während der Periode 1993-2000 beobachtet wurden. Unsere Ergebnisse zeigen auf, dass das Verschuldungsniveaus positiv korreliert mit der Verfügbarkeit der Aktiven und mit dem Wachstum, und negativ korreliert mit der Rentabilität. Diese Ergebnisse sind stark abhängig von der Unternehmungsgrösse. So sind 20 Mitarbeitern die strategische Schwelle für das finanzielle Verhalten der kleinen und mittleren französischen Unternehmen.

\section{Introduction}

La théorie financière moderne vise principalement à déterminer le niveau optimal d'endettement permettant de maximiser la valeur de l'entreprise. Les travaux de Modigliani et Miller (1958) ont permis de définir un cadre d'analyse théorique pertinent de l'incidence du niveau d'endettement sur la valeur de la firme. Leur 
démarche, basée sur l'hypothèse forte de la perfection des marchés financiers, montre le caractère parfaitement substituable des principaux modes de financement de l'investissement.

Progressivement, la prise en compte d'imperfections sur les marchés de capitaux, telles que l'incidence de la fiscalité, du risque de faillite ou des coûts d'agence, dans un cadre d'analyse marqué par le caractère asymétrique de l'information, a permis d'établir l'importance des facteurs financiers dans la décision d'investissement des entreprises ${ }^{1}$. Par la suite, ces analyses ont été complétées par deux approches plus pragmatiques : le financement par hiérarchie et par compromis. Ces éléments revêtent une importance particulière pour des entreprises de taille réduite, structurellement confrontées à des problèmes financiers spécifiques tels que l'accès limité aux ressources financières, un risque financier supérieur, une insuffisance de fonds propres ou une difficulté accrue de gestion des liquidités (St-Pierre, 1994).

Nous tentons de vérifier, à l'aide de techniques récentes d'analyse des données de panel, l'impact des variables relevées par ces théories sur le niveau d'endettement de 2880 PME françaises observées au cours de la période 19932000. Notre réflexion est organisée de la façon suivante : les éléments théoriques sont résumés dans une première section, les hypothèses testables dans une seconde. La section 3 expose les caractéristiques de la base de données et les variables retenues et l'analyse empirique est menée dans la section 4 .

\section{Les éléments théoriques}

Le modèle initial de Modigliani et Miller (1958), aboutissant à la proposition d'indifférence, est le point de départ des réflexions concernant la structure financière. Cette proposition, aux conditions de validité très restrictives, ne permet pas de tenir compte des conditions individuelles et concrètes de financement. Pour ce faire, nous présentons des théories basées principalement sur la remise en cause de l'hypothèse de perfection des marchés de capitaux.

\subsection{Avantage fiscal et coûts de faillite : un arbitrage nécessaire}

Les économies d'impôts liées au financement par emprunt favorisent ce mode de financement (Modigliani et Miller, 1963). Toutefois, l'imposition personnelle (Miller, 1977) ainsi que des procédés alternatifs de déductibilité fiscale (De Angelo et Masulis, 1980) nuancent le caractère par trop général du rôle supposé de la fiscalité dans la détermination de la structure d'endettement des firmes. De plus,

1. Pour une revue littéraire, se reporter à Harris et Raviv (1991); pour une approche empirique, voir Rajan et Zingales (1995). 
si la prise en compte d'éléments fiscaux induit une préférence pour la dette, celle-ci ne peut se concevoir sans augmentation conséquente des charges financières et donc du risque de défaillance pour des entreprises déjà structurellement exposées $^{2}$. Ainsi, la structure d'endettement optimale se définit comme la résultante d'un arbitrage individuel entre gain fiscal de l'endettement et coûts résultant d'une éventuelle faillite.

\subsection{Théorie de l'agence et PME}

La théorie de l'agence appliquée aux PME souligne le caractère incomplet des contrats liant le propriétaire-dirigeant aux bailleurs de fonds de l'entreprise, mais aussi aux autres acteurs de la vie de l'entreprise (famille, clients, personnel). Dans le cas d'institutions financières, ces imperfections sont notamment liées à des objectifs divergents pour lesquels les deux parties ne peuvent adopter un comportement similaire en raison d'un accès asymétrique à l'information : du côté offre, le manque d'information des créanciers influe sur la perception du risque encouru et donc sur la quantité, le coût et les restrictions liées aux crédits octroyés (sélection adverse); à l'inverse, les petites entreprises décrivent souvent l'offre bancaire comme inadaptée à la demande, tout en étant en mesure d'utiliser l'information pertinente à des fins personnelles (hasard moral).

\subsection{Un financement par «compromis » ou par « hiérarchie »?}

Les théories mettant en balance les avantages fiscaux de la dette avec les coûts de faillite et les conflits d'agence émergeant des rapports contractuels entre dirigeant et créanciers de l'entreprise ont permis l'élaboration d'une théorie de l'endettement optimal (Static Trade-off Theory ou STT). Cette théorie est basée sur un raisonnement marginaliste sous-entendant l'existence d'un ratio optimal d'endettement (Myers, 1984). À l'inverse, la théorie du financement hiérarchique (Pecking Order Theory ou POT) néglige l'existence d'un ratio cible d'endettement au profit d'une règle principale de financement prioritaire basée sur l'existence d'asymétries informationnelles durant la phase de financement de l'entreprise (Myers et Majluf, 1984). Cette hiérarchie peut toutefois s'exprimer différemment en fonction de l'objectif poursuivi par le dirigeant de l'entreprise (Bultel-Poincelot, 1994). Empiriquement, Shyam-Sunders et Myers (1999) valident l'hypothèse d'un financement hiérarchique au détriment d'une démarche basée sur la recherche d'un endettement optimal ${ }^{3}$.

2. Pour une présentation détaillée des coûts de faillite, se référer à Malécot (1984).

3. Pour une vision critique de ces résultats, voir Chirinko et Shinga (2000) et Frank et Goyal (2003). Se référer à Carpentier et Suret (2000) pour des tests concernant les grandes entreprises françaises. 


\section{Les hypothèses testées}

Partant des éléments théoriques discutés, l'objet de cette section consiste à décrire les hypothèses testées. Ces hypothèses concernent l'influence de variables liées aux caractéristiques de l'entreprise et considérées par la théorie comme des facteurs explicatifs du niveau d'endettement.

\subsection{La taille}

Les conditions d'accès aux sources de financement externes varient selon la taille des entreprises. En effet, les plus petites entreprises ont une probabilité de défaut plus importante et l'information disponible à leur sujet est sensiblement réduite, stigmatisant les conflits d'intérêt avec les créanciers. Par conséquent, la taille est supposée être un facteur explicatif important dans le comportement d'endettement des firmes de notre échantillon.

\subsection{L’âge}

Nous considérons la durée de vie de l'entreprise comme représentative du capital informationnel dont peuvent disposer les créanciers. À ce titre, elle influence la disponibilité du capital par le biais d'une réduction de l'incertitude relative aux capacités de remboursement. Nous testons donc l'hypothèse selon laquelle les entreprises plus âgées ont un endettement plus important.

\subsection{La rentabilité}

En référence aux arguments de la théorie du financement hiérarchique, les entreprises les plus rentables devraient se caractériser, toutes choses égales d'ailleurs, par un endettement moindre (Myers, 1977). À l'inverse, selon les tenants de la théorie du signal, les banques utilisant la rentabilité passée dans l'évaluation du risque des entreprises augmenteront la capacité d'emprunt des firmes les plus profitables (Ross, 1977). L'hypothèse testée consiste à confirmer l'une ou l'autre de ces théories dans le cas des PME françaises.

\subsection{La croissance}

La croissance est un élément moteur dans l'activité d'un nombre important de petites entreprises. Toutefois, des différences significatives apparaissent entre les firmes selon le niveau de croissance constaté (Hutchinson et Ray, 1986). À nouveau, deux thèses sont en concurrence, d'où l'hypothèse alternative : selon une approche privilégiant les coûts de faillite ou d'agence, les possibilités de croissance sont une source de conflits et donc de réduction du niveau d'endettement; à l'inverse, 
selon une perspective de financement hiérarchique, l'endettement apparaît comme un moyen efficace de financement et de préservation du contrôle pour le propriétaire-dirigeant de l'entreprise.

\subsection{La composition de l'actif}

La composition de l'actif est un élément décisif dans la décision d'octroi de crédits aux $\mathrm{PME}^{4}$. Les entreprises présentant des niveaux élevés d'actifs corporels sont en mesure de réduire les asymétries d'information et les coûts d'agence associés. Ainsi, l'importance des actifs corporels devrait se traduire par un endettement supérieur, toutes choses égales d'ailleurs.

\subsection{Le coût financier}

En dépit d'une préférence supposée pour la disponibilité, nous considérons l'impact du coût du financement par emprunt pour les PME. Ce coût, lié au risque de défaut, se traduit par un renchérissement du crédit pour les plus petites d'entre elles. Toutefois, selon St-Pierre (1994), cet argument peut permettre aux institutions financières de réaliser des profits supérieurs sur cette catégorie de clientèle. Nous supposons donc une relation négative entre le coût du financement et le niveau d'endettement des firmes.

\section{Les données et variables utilisées}

\subsection{Les données utilisées}

Les données collectées pour cette étude proviennent de la base de données DIANE (disque pour l'analyse économique) qui recensent les informations comptables, bilans et comptes de résultats d'entreprises françaises. La sélection de l'échantillon a été effectuée selon les critères suivants :

- données financières disponibles et mises à jour ;

- effectifs annuels inférieurs à 500 employés pour les années 1993-2000, ce seuil permettant de prendre en compte un maximum d'observations de PME;

- en raison des spécificités de gestion des firmes financières, publiques, cotées ainsi que celles dont les capitaux propres sont négatifs, ces firmes sont exclues.

4. Pour une revue littéraire du rôle des garanties, se référer à Berger et Udell (1998) ; voir Elsas et Krahnen (2000) pour une approche empirique. 
L'échantillon non cylindré, après nettoyage ${ }^{5}$, se compose de 2880 firmes observées durant une période de cinq à huit ans comme le montre la répartition par taille suivante.

TABLEAu 1

Répartition du panel par taille selon les effectifs moyens

\begin{tabular}{lcccccc}
\hline Années & $\begin{array}{c}\text { Total } \\
\text { firmes }\end{array}$ & $\begin{array}{c}\text { Classe } \\
\mathbf{0 - 9}\end{array}$ & $\begin{array}{c}\text { Classe } \\
\mathbf{1 0 - 1 9}\end{array}$ & $\begin{array}{c}\text { Classe } \\
\mathbf{2 0 - 4 9}\end{array}$ & $\begin{array}{c}\text { Classe } \\
\mathbf{5 0 - 9 9}\end{array}$ & $\begin{array}{c}\text { Classe } \\
\mathbf{1 0 0 - 4 9 9}\end{array}$ \\
\hline 1993 & 318 & 89 & 83 & 64 & 52 & 30 \\
1994 & 331 & 92 & 85 & 67 & 55 & 32 \\
1995 & 341 & 94 & 88 & 69 & 57 & 33 \\
1996 & 365 & 100 & 95 & 76 & 61 & 33 \\
1997 & 414 & 111 & 103 & 88 & 79 & 33 \\
1998 & 388 & 108 & 101 & 73 & 77 & 29 \\
1999 & 369 & 104 & 99 & 71 & 68 & 27 \\
2000 & 354 & 101 & 92 & 71 & 65 & 25 \\
\hline Total & $\mathbf{2 ~ 8 8 0}$ & $\mathbf{7 9 9}$ & $\mathbf{7 4 6}$ & $\mathbf{5 7 9}$ & $\mathbf{5 1 4}$ & $\mathbf{2 4 2}$ \\
\hline
\end{tabular}

\subsection{Les variables retenues}

Les variables sélectionnées sont extraites des états financiers des firmes de notre échantillon et choisies de façon à tester les différentes hypothèses établies. Les statistiques descriptives relatives à ces variables sont détaillées dans l'annexe I.

- La variable caractéristique de la structure d'endettement ou variable expliquée est la somme des dettes financières rapportées au total de l'actif. Le choix de l'actif total vise à neutraliser l'effet taille déjà pris en compte.

- La taille est mesurée par les effectifs moyens des firmes au cours de la période 1993-2000, d'où la construction de cinq classes de taille aux seuils suivants : (0-9), (10-19), (20-49), (50-99), (100-499).

- La durée de vie des firmes est mesurée, pour chaque année, par le biais d'une variable représentative de l'âge. Cet indicateur représente le capital informationnel existant sur l'entreprise.

- La performance des entreprises est mesurée par le ratio de rentabilité des fonds propres définit comme le résultat net rapporté aux fonds propres de l'entreprise.

5. L'échantillon a subi un nettoyage des valeurs aberrantes et extrêmes selon la méthode des intervalles interquartiles présentée par Kremp (1995), conduisant à l'élimination de $1,4 \%$ des observations. 
- L'indicateur de croissance est calculé par la variabilité du chiffre d'affaires net de l'entreprise d'une année à l'autre. Cette mesure, bien qu'imparfaite, doit permettre de quantifier l'évolution de l'activité des entreprises au cours des années considérées.

- Les données disponibles ne permettant pas de mesurer l'incidence des garanties personnelles sur les contrats de prêts, la structure de l'actif a pour but de renseigner sur le montant des collatéraux que l'entreprise peut offrir à ses créanciers. Le ratio utilisé est la somme des immobilisations corporelles nettes et des stocks rapportée au total de l'actif.

- Le coût apparent du financement est mesuré par la somme des intérêts payés rapportée au montant de la dette financière. Bien qu'imparfaite, cette variable est à notre sens la plus représentative de la valeur des coûts directs de l'endettement supportés par les firmes de notre échantillon.

\section{Analyses empiriques}

\section{1. Évolution du taux d'endettement durant la période 1993-2000}

Le graphique (figure 1) retrace l'évolution de l'endettement total (concours bancaires, dettes commerciales, prêts de groupe et associés) rapporté au total du bilan; nous commentons également les résultats de tests d'égalité des moyennes du ratio entre les séries chronologiques pour chaque classe de taille (t-tests).

Après un début de décennie marqué par un fort désendettement de la part de l'ensemble des entreprises du panel, la situation se stabilise pour les moyennes entreprises observées entre 1993 et 1995 : les moyennes du ratio ne sont pas statistiquement différentes pour les entreprises de plus de 20 salariés; en revanche, les plus petites firmes (moins de 20 salariés) connaissent une baisse significative, au seuil de $10 \%$, du ratio moyen d'endettement sur cette période. Cette situation peut s'expliquer par des niveaux initiaux plus importants, mais aussi par une attitude plus prudente, voulue ou subie, des plus petites firmes après une année 1993 marquée par un ralentissement important de l'activité française (crise de liquidité).

Au cours des années 1995-1998, l'endettement moyen diminue de façon significative pour toutes les catégories d'entreprises de l'échantillon, au seuil de $10 \%$, à l'exception de la classe $4(50<$ effectifs < 99). L'ensemble de la période d'observation 1993-1997 se caractérise par une relation décroissante et stable entre la taille et le niveau d'endettement des firmes. Cette relation est beaucoup moins prononcée pour la période 1998-2000 marquée par le retour d'une croissance soutenue de l'économie française. Durant ces deux années, la progression rapide de l'endettement, significative au seuil de $5 \%$ pour toutes les firmes, peut s'expliquer par 
FIGURE 1

\section{Ratio de l'endettement rapporté au total du bilan entre 1993-2000} (médiane par classe d'effectifs)

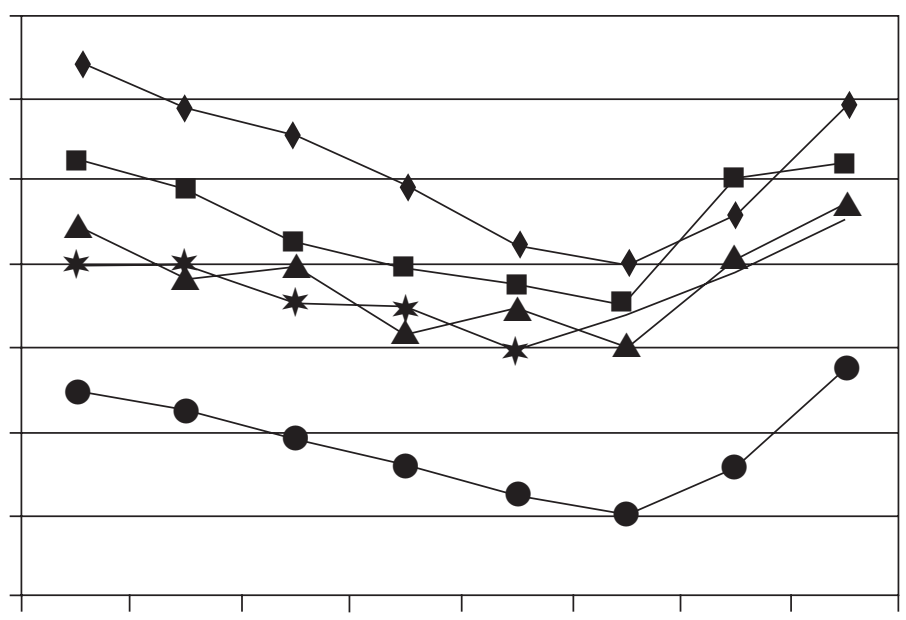

\section{\begin{tabular}{llllll}
\hline$-0-9$ & $-\boldsymbol{\square}-10-19$ & $-\boldsymbol{\Delta}-20-49$ & $-\star *-50-99$ & $-\mathbf{0}-99-499$
\end{tabular}}

un «effet croissance », mais également par la baisse des taux d'intérêt réels au cours de la période permettant de faire jouer un effet de levier important, particulièrement pour les grandes PME (effectifs > 99 employés).

\subsection{Analyse de la variance}

L'analyse de la décomposition de la variance, par le biais d'un modèle statique en niveau, a pour but de mesurer la contribution de chaque variable exogène dans la variance totale et expliquée de la variable endogène: le taux d'endettement financier.

Cette décomposition montre que, relativement à la période étudiée et aux éléments conjoncturels décrits ( $c f .4 .2)$, l'aspect temporel est un élément significatif de la variation du ratio d'endettement des entreprises observées (3,2\% de la variance totale). L'effet taille explique également une partie non négligeable de la variance totale $(4,8 \%)$, justifiant ainsi une analyse discriminée entre les entreprises selon ce critère. La rentabilité des firmes est, parmi les variables explicatives extraites du bilan, la variable apportant la contribution la plus significative $(10,8 \%)$. Ensuite, les variables de croissance $(3,6 \%)$ et de garanties $(2,7 \%)$ sont les éléments les 
TABLEAU 2

Analyse de la décomposition de la variance

\begin{tabular}{lcc}
\hline \multirow{2}{*}{ Variables explicatives } & \multicolumn{2}{c}{ Contribution de chaque variable explicative (en \%) } \\
\cline { 2 - 3 } & de la variance totale & de la variance expliquée \\
\hline Dimension temporelle & 3,2 & 14,2 \\
Taille & 4,8 & 15,1 \\
Âge & 0,5 & 1,4 \\
Rentabilité & 10,8 & 39,4 \\
Croissance & 3,6 & 15,5 \\
Garanties & 2,7 & 9,8 \\
Coût du financement & 1,2 & 4,6 \\
\hline Variance expliquée totale & $\mathbf{2 6 , 8}$ & $\mathbf{1 0 0}$ \\
\hline
\end{tabular}

plus pertinents tandis que les indicateurs de coût du financement $(1,2 \%)$ et de durée de vie $(0,5 \%)$, n'intégrant qu'une partie de l'information existante par le biais de mesures approchantes, n'apportent qu'une contribution marginale au modèle de décomposition de la variance.

\subsection{La méthode d'estimation}

Après l'analyse de l'évolution du taux d'endettement et de la décomposition de la variance durant la période considérée, la dernière étape de notre recherche consiste à vérifier économétriquement l'impact des variables explicatives de la structure d'endettement des petites et moyennes entreprises françaises.

L'étude de données issues de bilans d'entreprises s'effectue selon les méthodes de l'économétrie de panel. Les méthodes traditionnelles largement utilisées regroupent les estimateurs «intra », «inter» et des moindres carrés quasi généralisés ${ }^{6}$. Toutefois, ces estimateurs ne sont pas convergents pour une période de temps finie notamment en raison des problèmes d'endogénéité des variables explicatives. Les progrès de l'économétrie ont permis, sous l'influence des travaux d'Arrelano et Bond (1991), d'appliquer la technique des moments généralisés. Cette technique d'estimation dynamique de la fonction d'endettement en deux étapes fait un meilleur usage de l'information disponible : dans une première étape, les variables potentiellement corrélées avec le terme d'erreur sont instrumentées de façon à neutraliser l'impact de l'endogénéité de certaines variables exogènes du modèle ; dans une seconde étape, le vecteur des résidus estimés est utilisé pour obtenir un estimateur asymptotiquement convergent. La validité de la combinaison d'instruments utilisés est validée au moyen du test de sur-identification de Sargan.

6. Pour une présentation de ces méthodes, consulter Dormont (1989).

Revue internationale P.M.E., vol. 17, $\mathrm{n}^{\circ}$ 1, 2004 
La statistique $m_{2}$ est calculée pour tester l'autocorrélation des perturbations du modèle en différences premières.

Le modèle testé est le suivant:

$$
\begin{aligned}
& \text { END } \left._{\text {Firme }_{\mathrm{I}}} \text { Année }_{\mathrm{T}}\right]=\alpha+\beta_{1} \mathrm{END}_{[\mathrm{I} ; \mathrm{T}-1]}+\beta_{3} \operatorname{AGE}_{[\mathrm{I} ; \mathrm{T}]}+\beta_{4} \operatorname{RENT}_{[\mathrm{I} ; \mathrm{T}]} \\
& +\beta_{5} \operatorname{CROISS}_{[\mathrm{I} ; \mathrm{T}]}+\beta_{6} \mathrm{GAR}_{[\mathrm{I} ; \mathrm{T}]}+\beta_{7} \mathrm{CTFIN}_{[\mathrm{I} ; \mathrm{T}]} \\
& +\eta_{[\mathrm{I}]}+\omega_{[\mathrm{T}]}+\varepsilon_{[\mathrm{I} ; \mathrm{T}]}
\end{aligned}
$$

Le coefficient retardé d'endettement $\left(E N D_{[I ; T-1]}\right)$, nécessaire aux estimations, représente un indicateur des coûts d'ajustement dans la description du comportement d'endettement des entreprises du panel. Un effet fixe individuel $\eta_{[]]}$et un effet temporel $\omega_{[\mathrm{T}]}$ sont ajoutés, le terme $\varepsilon_{[\mathrm{I} ; \mathrm{T}]}$ matérialisant le vecteur des résidus ; l'influence sectorielle est prise en compte au niveau 17 de la nomenclature d'activités française par le biais de variables indicatrices ${ }^{7}$.

\subsection{Les estimations de la fonction d'endettement}

Les résultats des estimations de la fonction d'endettement sont présentés dans le tableau 3. Les deux modèles testés correspondent à des estimateurs des moments généralisés selon Arrelano et Bond (1991) pour lesquels l'utilisation de retards dans l'instrumentation est sensiblement différente. Toutefois, les coefficients obtenus sont assez proches et, dans les deux cas, justifient l'utilisation de variables explicatives du comportement d'endettement des firmes par des degrés de significativité élevés. Les statistiques de tests de Sargan et d'autocorrélation $\left(m_{2}\right)$ permettent de valider la combinaison d'instruments utilisés et de rejeter la présence d'autocorrélation à l'ordre 2, le modèle étant en différences premières.

\subsection{Interprétation des résultats}

L'influence statistique de la variable d'endettement retardé souligne le caractère dynamique de la fonction d'endettement des firmes de l'échantillon. Elle permet également une mise en perspective dans le cadre d'une approche en termes de ratio cible puisque ces coefficients traduisent l'existence de coûts d'ajustement du niveau d'endettement, notamment liés à l'incidence de facteurs macroéconomiques.

La variable représentative de la durée de vie des entreprises n'est clairement significative, au seuil de $10 \%$, que pour les firmes appartenant aux deux premières classes de taille (moins de 20 salariés). Cette mesure, dont l'objectif est de quantifier à la fois le capital informationnel détenu par les créanciers de la firme et la capacité

7. Les secteurs $\mathbf{J}$ (activités financières) et $\mathrm{L}$ (administration publique) sont par définition exclus. Pour plus de détails, voir l'annexe II. 


\section{TABLEAU 3}

Résultats des estimations dynamiques de la fonction d'endettement Période 1993-2000

\begin{tabular}{|c|c|c|c|c|c|c|c|}
\hline Variables & $\begin{array}{l}\text { Ensemble } \\
\text { Modèle } 1\end{array}$ & $\begin{array}{l}\text { Ensemble } \\
\text { Modèle } 2\end{array}$ & $\begin{array}{c}\text { Classe } 1 \\
(0-9)\end{array}$ & $\begin{array}{c}\text { Classe } 2 \\
(10-19)\end{array}$ & $\begin{array}{c}\text { Classe } 3 \\
(20-49)\end{array}$ & $\begin{array}{c}\text { Classe } 4 \\
(50-99)\end{array}$ & $\begin{array}{c}\text { Classe } 5 \\
(100-499)\end{array}$ \\
\hline Endettement $(-1)$ & $\begin{array}{c}0,615^{*} \\
(0,068)\end{array}$ & $\begin{array}{c}0,628^{*} \\
(0,057)\end{array}$ & $\begin{array}{c}0,525^{*} \\
(0,062)\end{array}$ & $\begin{array}{c}0,567 * \\
(0,061)\end{array}$ & $\begin{array}{c}0,617^{*} \\
(0,054)\end{array}$ & $\begin{array}{c}0,654 * \\
(0,058)\end{array}$ & $\begin{array}{c}0,612^{*} \\
(0,055)\end{array}$ \\
\hline Âge & $\begin{array}{c}0,041 \\
(0,028)\end{array}$ & $\begin{array}{l}0,027 \text { *** } \\
(0,015)\end{array}$ & $\begin{array}{l}0,016 \text { **** } \\
(0,009)\end{array}$ & $\begin{array}{l}0,044 * * * \\
(0,025)\end{array}$ & $\begin{array}{c}0,031 \\
(0,025)\end{array}$ & $\begin{array}{c}0,027 \\
(0,022)\end{array}$ & $\begin{array}{c}0,039 \\
(0,027)\end{array}$ \\
\hline Rentabilité & $\begin{array}{r}0,389^{*} \\
(0,053)\end{array}$ & $\begin{array}{c}-0,315^{*} \\
(0,048)\end{array}$ & $\begin{array}{c}-0,291^{*} \\
(0,045)\end{array}$ & $\begin{array}{r}0,352^{*} \\
(0,049)\end{array}$ & $\begin{array}{c}-0,336^{*} \\
(0,047)\end{array}$ & $\begin{array}{r}-0,410^{*} \\
(0,058)\end{array}$ & $\begin{array}{c}-0,397^{*} \\
(0,051)\end{array}$ \\
\hline Croissance & $\begin{array}{l}0,058^{* * * *} \\
(0,033)\end{array}$ & $\begin{array}{l}0,049 \text { ** } \\
(0,023)\end{array}$ & $\begin{array}{c}0,069 \\
(0,047)\end{array}$ & $\begin{array}{l}0,108 \text { *** } \\
(0,058)\end{array}$ & $\begin{array}{l}0,098^{* *} \\
(0,045)\end{array}$ & $\begin{array}{l}0,045^{* * *} \\
(0,019)\end{array}$ & $\begin{array}{l}0,061 * * \\
(0,028)\end{array}$ \\
\hline Garanties & $\begin{array}{l}0,258^{* * *} \\
(0,116)\end{array}$ & $\begin{array}{c}0,301^{*} \\
(0,075)\end{array}$ & $\begin{array}{r}0,327^{*} \\
(0,072)\end{array}$ & $\begin{array}{c}0,314^{*} \\
(0,076)\end{array}$ & $\begin{array}{l}0,281 \text { ** } \\
(0,133)\end{array}$ & $\begin{array}{l}0,401 \text { *** } \\
(0,221)\end{array}$ & $\begin{array}{c}0,287 * * \\
(0,129)\end{array}$ \\
\hline $\begin{array}{l}\text { Coût du } \\
\text { financement }\end{array}$ & $\begin{array}{c}-0,027 * * \\
(0,012)\end{array}$ & $\begin{array}{c}-0,037 * * \\
(0,014)\end{array}$ & $\begin{array}{c}-0,031 \text { *** } \\
(0,016)\end{array}$ & $\begin{array}{c}-0,028 \text { *** } \\
(0,015)\end{array}$ & $\begin{array}{c}-0,057 * * \\
(0,024)\end{array}$ & $\begin{array}{c}-0,040^{*} \\
(0,011)\end{array}$ & $\begin{array}{c}-0,019^{*} \\
(0,06)\end{array}$ \\
\hline Sargan & 11,245 & 13,714 & 8,109 & 12,188 & 7,645 & 9,371 & 7,551 \\
\hline$m_{2}$ & $-0,341$ & $-0,294$ & $-0,245$ & $-0,473$ & $-0,974$ & $-0,511$ & $-0,214$ \\
\hline $\mathrm{N}$ & 2880 & 2880 & 799 & 746 & 579 & 514 & 242 \\
\hline
\end{tabular}

Écarts types robustes entre parenthèses $*: p \leq 0,1, * *: p \leq 0,05, * * *: p \leq 0,01$.

Modèles estimés en différences premières avec indicatrices temporelles, sectorielles et constantes.

Les instruments sont en niveaux, les estimations par taille sont identiques au modèle 2.

Modèle 1 : variable endogène retardée $t-3$ et $t-4$, variables exogènes retardées $t-2$, sauf Âge: exogène.

Modèle 2: variable endogène retardée $t-3$ et $t-4$, variables exogènes retardées $t-2$, sauf Rentabilité : retardée $t-2$ et $t-3$ et Âge : exogène.

pour cette dernière de bénéficier d'un autofinancement conséquent, n'est donc que peu valide dans notre cas. Cela peut notamment s'expliquer par la prise en compte d'un critère de rentabilité dans nos estimations, relativisant l'influence de la réputation à une date antérieure à 1993 pour la majorité des firmes. De plus, comme le montrent Petersen et Rajan (1994) sur un échantillon de PME américaines, l'impact de la durée de vie n'est pas linéaire: très significatif dans les premières années, il décroît fortement par la suite. Ainsi, les firmes les plus petites de notre échantillon, qui sont également les plus jeunes ${ }^{8}$, affichent une sensibilité (positive) plus importante que les autres au critère de l'âge dans la détermination du niveau d'endettement.

8. Nous relevons que $28 \%$ des firmes de la classe de taille 1 ont moins de huit ans d'existence, ce pourcentage chutant à $25 \%, 21 \%, 15 \%$ et $9,5 \%$ respectivement pour les classes $2,3,4$ et 5 . 
La variable de rentabilité est corrélée négativement à l'endettement pour toutes les firmes de notre panel avec un seuil de significativité élevé (1\%). Ce résultat, contraire aux prédictions théoriques liées à la théorie du signal (Ross, 1977), valide, dans une certaine mesure puisque c'est le cas pour toutes les catégories de taille, les principes de l'existence d'une hiérarchie des financements décrite par Myers (1984) et s'appliquant au cas des PME françaises.

Comme le suppose l'approche hiérarchique de la théorie du financement, la variable matérialisant la croissance des firmes influe de façon positive et significative sur le montant d'endettement des entreprises. Toutefois, à travers le découpage par taille, l'effet n'est pas symétrique. Le seuil de 20 salariés apparaît comme un minimum à partir duquel la variation du chiffre d'affaires joue un rôle prépondérant, comme élément de signalisation, dans l'expression de la capacité d'endettement des PME observées. Pour les firmes comptant moins d'une vingtaine de salariés, cet effet croissance reste très faible : il est juste significatif pour la classe de taille 2 (10-19 salariés) au seuil de $10 \%$ et non significatif en deçà (0-10).

Le montant des garanties figurant aux comptes des entreprises est un élément positivement corrélé à l'endettement. La variable Garanties est significative au seuil de $1 \%$ pour les entreprises dont les effectifs sont inférieurs à 20 salariés, au-delà, le degré de significativité des coefficients est moindre (5\%). La tangibilité des actifs est donc prioritaire pour les entreprises les plus petites et les plus jeunes en raison d'asymétries informationnelles, vis-à-vis des créanciers, supérieures. Ce résultat confirme les prédictions de la théorie de l'agence selon lesquelles la capacité de ces entreprises à garantir leurs emprunts est un élément déterminant dans la décision que prennent les créanciers d'octroyer des crédits.

Les coefficients du coût du financement indiquent que la demande de crédit des entreprises est négativement reliée à son coût financier, mais cela de façon inégale selon la taille. Les firmes les plus importantes, appartenant aux classes de taille 4 et 5, se caractérisent par une sensibilité supérieure aux coûts de l'emprunt. Ces résultats soulignent que les grandes PME sont en mesure de bénéficier de sources de financement alternatives et d'économies d'impôts non liées à la dette plus importante. À l'inverse, pour les plus petites entreprises, souvent confrontées à des problèmes de disponibilité du capital, l'aspect coût semble secondaire puisqu'il n'est que faiblement significatif (10\%) pour les firmes de moins de 20 salariés.

\section{Conclusion}

En résumé, il apparaît qu'il n'y a pas indifférence quant au choix de la structure d'endettement des petites et moyennes entreprises françaises observées. À l'inverse, nos estimations confirment que les problèmes d'asymétrie de l'information jouent 
un rôle central dans la détermination d'une structure d'endettement optimale. Les variables relevées par les théories des coûts de faillite, d'agence et du financement hiérarchique sont pertinentes d'un point de vue empirique. Toutefois, les comportements diffèrent sensiblement en fonction du critère de taille puisque les indicateurs de croissance (influence positive) et de coût du financement (influence négative) sont particulièrement significatifs dans le choix des firmes les plus grandes de l'échantillon (plus de 20 salariés). Inversement, la durée de vie (influence positive) ainsi que l'importance des actifs immobilisés (influence positive) sont d'autant plus significatifs que la taille des firmes est réduite (moins de 20 salariés). Seul le critère de rentabilité, confirmant l'existence d'une hiérarchie des financements, influe de façon négative et assez tranchée sur l'endettement des 2880 firmes observées. Ces résultats montrent qu'il existe, au sein de la catégorie des PME françaises, des déterminants similaires du niveau d'endettement, mais d'importance inégale selon la taille des firmes considérées.

ANNEXE I

Statistiques descriptives des variables en 1996

\begin{tabular}{lccccc}
\hline Variables & Moyenne & Médiane & Écart type & Minimum & Maximum \\
\hline Endettement financier & 0,31 & 0,30 & 0,17 & 0,00 & 0,89 \\
Âge & 13,22 & 11 & 3 & 5 & 97 \\
Rentabilité & 0,06 & 0,05 & 0,03 & $-2,05$ & 0,71 \\
Croissance & 6,84 & 5,23 & 2,58 & $-12,26$ & 74,97 \\
Garanties & 0,47 & 0,42 & 0,12 & 0,01 & 0,98 \\
Coût du financement & 7,01 & 6,45 & 2,25 & 0 & 28,19 \\
Effectifs & 18 & 16 & 10 & 0 & 497 \\
\hline
\end{tabular}


ANNEXe II

Ventilation du panel par secteur d'activité

\begin{tabular}{lc}
\hline Secteurs & Nombre \\
Nomenclature d'activités française - Niveau 17 & de firmes \\
\hline Agriculture, chasse et sylviculture & 24 \\
Pêche, aquaculture, services annexes & 37 \\
Industries extractives & 117 \\
Industrie manufacturière & 593 \\
Production et distribution d'électricité, de gaz et d'eau & 45 \\
Construction & 419 \\
Commerce, réparations automobiles et articles domestiques & 477 \\
Hôtels et restaurants & 247 \\
Transports et communications & 284 \\
Activités financières & 0 \\
Immobilier, location et services aux entreprises & 199 \\
Administration publique & 0 \\
Éducation & 70 \\
Santé et action sociale & 82 \\
Services collectifs, sociaux et personnels & 128 \\
Activité des ménages & 94 \\
Activités extraterritoriales & 64 \\
Total & $\mathbf{2 8 8 0}$ \\
\hline
\end{tabular}

\section{Bibliographie}

ARrelano, M. et S. Bond (1991), «Some tests of specification for panel data: Monte Carlo evidence and an application to employment equations », Review of Economic Studies, vol. 58, nº 2, p. 277-297.

BERGER, A.N. et G.F. UdELL (1998), «The economics of small business finance: the roles of private equity and debt markets in the financial growth cycle », Journal of Banking and Finance, vol. 22, n ${ }^{\circ}$ 6, p. 613-673.

Bultel-PoinCELOT, E. (1994), Théories du financement hiérarchique et structure financière de la firme, Thèse de doctorat, Université de Bourgogne.

CARPEnTiER, C. et J.M. SuRET (2000), «Stratégies de fnancement des entreprises françaises : une analyse empirique », Finance, vol. 21, no 1, p. 9-34.

CHIRINKo, R.S. et A.R. SingHA (2000), « Testing static trade-off against pecking order models of capital structure : a critical comment », Journal of Financial Economics, vol. $58, \mathrm{n}^{\circ} 3$, p. 417-425.

De Angelo, H. et R. Masulis (1980), «Optimal capital structure under corporate and personal taxation », Journal of Financial Economics, vol. 8, n 3, p. 3-29. 
DORMONT, B. (1989), Introduction à l'économétrie des données de panel, Paris, Éditions CNRS.

ELSAS, R. et J.P. KRAHNEN (2000), «Collateral, default risk and relationship lending : an empirical study on financial contracting », Cahier de recherche $n^{\circ} 2540$, Frankfort, Center for Financial Studies.

FrANK, M. et V. GOYAL (2003), «Testing the pecking order theory of capital structure », Journal of Financial Economics, vol. 67, n 2, p. 217-248.

HARRIS, M. et A. RAVIV (1991), «The theory of capital structure», Journal of Finance, vol. 46, n 1 , p. 297-355.

HUTChinson, P. et G. RAY (1986), «Surviving the financial stress of small enterprise growth », dans J. Curran, J. Stanworth et D. Watkins (dir), The Survival of Small Firms, vol. 1: The Economics of Survival and Entrepreneurship, Aldershot, Angleterre, Gower Publishing.

KREMP, E. (1995), « Nettoyage des fichiers dans le cas de données individuelles », Économie et prévision, $\mathrm{n}^{\circ} 119$, p. 171-193.

MALÉCOT, J.F. (1984), «La mesure empirique des coûts de faillite: le cas français », Finance, vol. 2, $\mathrm{n}^{\circ}$ 1, p. 209-219.

MiLLER, M. (1977), «Debt and taxes », Journal of Finance, vol. 32, n 2, p. 261-275.

Modigliani, F. et M.H. Miller (1958), «The cost of capital, corporation finance and the theory of investment», American Economic Review, vol. 48, n 3, p. 261-297.

Modigliani, F. et M.H. Miller (1963), « Corporate income taxes and the cost of capital: a correction », American Economic Review, vol. 53, n 3, p. 433-443.

MYERS, S.C. (1977), «Determinants of corporate borrowing », Journal of Financial Economics, vol. 5, no 2, p. 147-175.

MYERS, S.C. (1984), «The capital sructure puzzle», Journal of Finance, vol. 39, n 3 , p. $575-592$.

MYERS, S.C. et N.S. MAJLUF (1984), «Corporate financing and investment decisions when firms have information that investors do not have», Journal of Financial Economics, vol. 13, no 2, p. 187-221.

PETERSEN, M.A. et R.G. RAJAN (1994), «The benefits of lending relationships : evidence from small business data », Journal of Finance, vol. 49, nº 1, p. 3-37.

RAJAN, R.G. et L. ZINGALES (1995), «What do we know about capital structure? Some evidence from international data », Journal of Finance, vol. 50, nº 6, p. 1421-1460.

Ross, S.A. (1977), «The determination of financial structure: the incentive-signaling approach », Bell Journal of Economics, vol. 8, n 1, p. 23-40.

SHYAM-SundERS, L. et S.C. MYERS (1999), «Testing static trade-off against pecking order models of capital structure », Journal of Financial Economics, vol. 51, $\mathrm{n}^{\circ} 2$, p. 219-244.

ST-PIERRE, J. (1994), «Les problèmes financiers des PME : l'état de la question», dans P.A. Julien (dir.), Les PME. Bilan et Perspectives, Paris, Economica. 